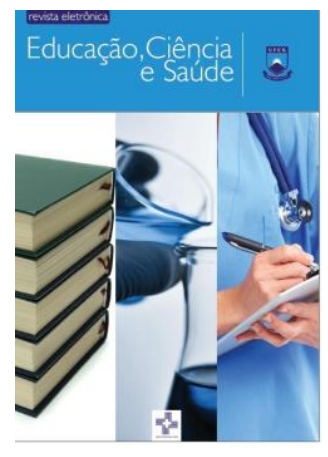

EDUCAÇÃO CIÊNCIA E SAÚDE

http://dx.doi.org/10.20438/ecs.v8i1.362

\title{
FERRAMENTAS SOCIOAMBIENTAIS EM PROL DA SAÚDE: A IMPORTÂNCIA E O ESTÍMULO DE BONS HÁBITOS DE HIGIENE EM TEMPOS DE COVID-19
}

\author{
André Luiz Costa Bósio1, Camila Mamede Damin'1, Fabiana Andreia Padia \\ Maniçoba $^{2}$, Vivian Almeida Assunção ${ }^{3}$, Rosani do Carmo de Oliveira Arruda ${ }^{3}$, \\ Flávia Maria Leme ${ }^{3}$ \\ ${ }^{1}$ Faculdade de Odontologia - FAODO, Universidade Federal de Mato Grosso do Sul - UFMS, \\ Campo Grande-MS, Brasil. \\ ${ }^{2}$ Faculdade de Ciências Farmacêuticas, Alimentos e Nutrição - FACFAN, Universidade Federal \\ de Mato Grosso do Sul - UFMS, Campo Grande-MS, Brasil. \\ 3Instituto de Biociências - INBIO, Universidade Federal de Mato Grosso do Sul - UFMS, \\ Campo Grande-MS, Brasil. \\ Email para correspondência: flavia.maria@ufms.br
}

\begin{abstract}
Resumo
O hábito de realizar uma boa higiene pessoal é iessencial para a qualidade de vida, pois auxilia na prevenção de doenças e no aumento da autoestima do indivíduo. Desse modo, objetivou-se sensibilizar crianças e adolescentes em risco social sobre a importância da lavagem das mãos para prevenção de doenças, principalmente em tempos de pandemia da COVID-19. Este trabalho é um relato de experiência da oficina de higiene pessoal desenvolvida durante o projeto de Extensão Ferramentas Socioambientais em Prol da Saúde, da Universidade Federal de Mato Grosso do Sul. A oficina de Higiene Pessoal foi realizada no Projeto Social Escolinha Tia More, situado no bairro Jardim Canguru, Campo Grande - MS, Brasil. Aplicou-se atividades lúdicas com músicas tocadas no violão, dinâmicas com as crianças e participantes envolvidos, jogos e brincadeiras para explicar noções relativas à higiene pessoal. Ao final da oficina, todos ganharam sabonetes e esponjas para o uso pessoal diário. As atividades realizadas geraram interatividade, estimulando a curiosidade sobre o tema.
\end{abstract}

Palavras-chave: higiene pessoal, material lúdico, cuidados pessoais, educação, saúde.

\section{Abstract}

The habit of performing good personal hygiene is essential for the better quality of life, because it helps in the prevention of diseases and in increasing the individual self-esteem. Thus, the objective was to sensitize children and adolescents at social 
risk about the importance of hand washing for disease prevention, especially in times of the COVID-19 pandemic. This work is an experience report of the Personal Hygiene workshop developed during the "Ferramentas Socioambientais em Prol da Saúde" project, from the Universidade Federal de Mato Grosso do Sul. The Personal Hygiene workshop was held at Projeto Social Escolinha Tia More, located in the neighborhood Jardim Canguru, Campo Grande - MS, Brazil. Playful activities were used, such as playing acoustic guitar, dynamics with the children and participants involved, and games to explain notions related to bathing and hand hygiene. At the end of the workshop, everyone received soaps and sponges for daily personal use. The activities carried out generated interactivity, stimulating curiosity about the theme.

Keywords: personal hygiene, playful material, personal care, education, health.

\section{Introdução}

No Brasil, a saúde e a educação são os dois pilares básicos garantidos pela Constituição Federal (Art. 6º, BRASIL, 1988) e que devem ser providos pelo estado gratuitamente. Para a implementação desse direito, os governos nos diferentes níveis hierárquicos contam com os recursos obtidos de impostos pagos pela população, de modo a proporcionar o mínimo de qualidade de vida a todos. Contudo, a população abaixo da linha da pobreza, 25,8\% (13,5 milhões) não têm acesso a esgoto e abastecimento de rede de água, e 21,1\% (11,1 milhões) não têm coleta de lixo (IBGE, 2019), o que aponta um ambiente favorável para a ocorrência de doenças que podem levar à morte.

Perante esse cenário, iniciativas de promoção da saúde por meio da educação, mais especificamente, de higiene profilática, são essenciais para a prevenção de doenças parasitárias. A falta de higiene básica com as mãos e/ou com a manipulação dos alimentos contribuem na contaminação e no ciclo de parasitas que afetam diretamente a saúde dos indivíduos que consomem esses alimentos (FERREIRA, 2008). Somado a isso, importante a ampliação do saneamento básico com critérios que incluam a conservação e preservação ambiental, especialmente dos recursos hídricos (Leoneti et al., 2011), pois as enterobioses são essencialmente provenientes do consumo de água contaminada.

A falta de informações, ou a inserção em prática, de técnicas relacionadas a higiene básica e com as mãos e/ou com a manipulação dos alimentos contribuem na contaminação e no ciclo de parasitas que afetam diretamente a saúde dos indivíduos que consomem esses alimentos (FERREIRA, 2008). 
Adicionado a este fato, podemos citar as condições precárias de habitação, geralmente longe dos serviços de oferta de redes de água e esgotamento sanitário, em que parte dos indivíduos em situação de vulnerabilidade social vivem, e geralmente desconhecem os riscos do ambiente nos quais estão inseridos. Essa situação tem sido agravada quando se trata de crianças em idade escolar, potencializando a transmissibilidade de doenças infecciosas e parasitárias, seja por bactérias, fungos e/ou vírus. A pandemia de Coronavírus (Covid-19) é o mais recente exemplo de desequilíbrio do processo saúdedoença. Há indícios de seu início na cidade chinesa de Wuhan no final de 2019, e expandiu-se por todo o mundo e no Brasil a partir do primeiro trimestre de 2020 (FARIAS, 2020).

O Ministério da Saúde declarou em 20 de março de 2020 que a Covid-19 passou a ser considerada uma doença de transmissão comunitária em todo o território nacional, ou seja, a ocorrência de novos casos deixou de ter ligação direta com os casos importados (BRASIL, 2020; CAVALCANTE, NETO et al., 2020). Nesse contexto, também não se pode ignorar que determinantes sociais têm grande impacto para o avanço da pandemia, expondo indivíduos que já vivem em vulnerabilidade social e econômica a uma maior transmissão e letalidade nos casos.

Segundo Oliveira et al. (2020, p. 4): "A transmissão do Sars-CoV-2 de pessoa para pessoa se dá por meio da auto inoculação do vírus em membranas mucosas (nariz, olhos ou boca) e do contato com superfícies inanimadas contaminadas." Assim, entende-se por Sars-Cov-2 como o nome da espécie de vírus infeccioso causador da doença e pandemia COVID-19 (ZHOU, et al., 2020; ZHU, et al., 2020).

A saúde e a educação são grandes pilares de manutenção e de continuidade da vida do ser humano, os quais vivem em eterna construção e desconstrução. Entretanto, a evolução da sociedade em um compromisso ético mostra que é possível promover saúde escolar através da troca de experiências entre professores, acadêmicos e a comunidade de modo interdisciplinar, do conhecimento adquirido nas universidades, e dos educadores envolvidos em contínuo processo crítico de saberes e práticas de saúde escolar a ser transmitido (FERRIANI, 1997). 
Dessa forma, neste período de pandemia relacionado à COVID-19, cuja prevenção consiste no reforço às práticas de limpeza de superfícies e de lavagem de mãos com sabonete e uso de álcool etílico $70 \%$, que servem como barreiras que visam impedir a contaminação e a propagação da doença.

Esses hábitos têm sido enfatizados e abordados como formas de se evitar uma maior propagação desse vírus, tanto em escolas como em mídias sociais, o que corrobora com a importância de inserção de projetos tanto no meio formal quanto informal, cujo objetivo inclua a profilaxia de doenças (KHATOON et al., 2017; ROCHA E SILVA, 2018; SOUZA-NETO et al., 2020). Além disso, uma alimentação saudável é um fator vital para manter a imunidade alta e reduzir a probabilidade do adoecimento.

Algumas ferramentas podem ser utilizadas para sensibilizar, a exemplo, em uma creche municipal de Garanhuns (PE), onde os professores utilizaram recursos didáticos (atividades orais, atividades escritas e jogos) e levantamento do conhecimento prévio dos alunos sobre higiene pessoal para sensibilizar as crianças a relacionarem a higiene pessoal com o meio em que vivem e contribuir com mudanças em hábitos de falta de higiene pessoal do cotidiano delas (ROCHA; SILVA, 2018).

Em uma ação acadêmica realizada com crianças do projeto de reforço escolar em Maceio ( $A L$ ), em que crianças em vulnerabilidade social foram sensibilizadas de forma lúdica sobre a importância de hábitos de higiene como: lavagens de mãos e de alimentos, andar calçado, e ter cautela com dejetos humanos e de animais, assim como abandonar hábitos desfavoráveis à conservação de sua saúde" (SOUZA-NETO et al., 2020). Em ambas as atividades os autores relataram terem êxito no desenvolvimento.

Assim, no contexto do aprendizado aliado à inserção de novos hábitos de higiene corporal, é fundamental para diminuir as taxas de transmissão de doenças, tais como: diarreia, doença de pele, infestações de verminoses e doenças bucais (KHATOON et al., 2017).

Vale ressaltar que as entoparasitoses não são notificadas compulsoriamente, logo não temos a real frequência delas na comundiade (TEIXEIRA et al., 2020). Por isso, o tema deve ser discutido em ambiente formal e informal em que as crianças/adolescentes estudam, pois além de receptivas 
ao aprendizado e novos hábitos, podem se tornar agentes de mudança e também ao divulgar na escola e entre seus familiares, podem transformar as suas comunidades em relação a qualidade de vida e de saúde (KHATOON et al., 2017).

Aliado a essa mesma ideia, atividades de extensão desenvolvidas pelas Instituições Federais de Ensino podem contribuir com a sensibilização da comunidade tanto acadêmica quanto a não acadêmica (LEME et al., 2015; BORTOLOTTO et al., 2016), pela troca de experiências.

Em 2018, 77\% da rede de esgoto de Campo Grande, capital do Estado de Mato Grosso Sul, passava pela canalização de esgoto, mas apenas 58,38\% eram processadas (CAMPO GRANDE-NEWS, 2008). Na Zona Sul de Campo Grande, a favela Cidade de Deus foi construída entre 2008 e 2009 . Fica ao lado do antigo lixão da cidade, erguida próximo ao antigo lixão da cidade entre os anos de 2008 e 2009, foi ocupada por famílias em vulnerabilidade social, muitos sobrevivendo somente da coleta e venda de resíduos sólidos recicláveis obtidos no antigo lixão (WIRTI; BERNARDELLI, 2016).

Em 2011, a Prefeitura de Campo Grande entregou várias casas distribuídas em quatro áreas de bairros diferentes em Campo Grande para a retirada dos moradores das proximidades do lixão que foi desativado. As casas ficam nos bairros: Vespasiano Martins, Jardim Canguru, José Teruel II (Dom Antônio Barbosa) e Bom Retiro (CAMPO GRANDE-NEWS, 2017). O Jardim Canguru recebeu em torno de 58 famílias. No Bairro, foi montada também a "Escolinha Tia More" pela moradora da antiga favela, Edileuza Luiz.

A escolinha é um projeto social, sem fins lucrativos, que atende 60 crianças (filhos de moradores) do Jardim em vulnerabilidade social. Na escolinha são realizadas atividades de leitura, canto e brincadeiras, contudo, o principal foco é proporcionar as principais refeições (almoço, lanche e janta) às crianças atendidas. A "Escolinha Tia More" se mantém somente com recursos oriundos de doação, ou seja, direcionados principalmente à alimentação das crianças.

Com essa preocupação, o Projeto de Extensão "Ferramentas Socioambientais em Prol da Saúde" desenvolveu, diversas oficinas voltadas para a prevenção da saúde e de cuidados com o meio ambiente. Dentre as Oficinas, destacamos a intitulada "Higiene Pessoal" que teve por objetivo 
sensibilizar crianças, adolescentes e adultos sobre a importância de cuidados básicos voltados à higiene corporal e, incrementando as medidas de prevenção à COVID-19 no Projeto Social Escolinha Tia More. O nosso país tem grande parte de sua população em situação de vulnerabilidade social, com muitos casos de adoecimento e morte por falta de informações básicas sobre o autocuidado. Assim, nosso objetivo aqui é relatar a experiência da execução da oficina de Higiene Pessoal, a fim de sensibilizar formadores de outras instituições a promoverem projetos com esse intuito.

\section{Metodologia}

Esse trabalho se caracteriza como um relato de experiência da oficina de higiene pessoal do Projeto de Extensão Ferramentas Socioambientais em Prol da Saúde realizado pela Universidade de Mato Grosso do Sul no Projeto Social Escolinha Tia More, realizada no dia 23 de outubro de 2020.

\subsection{Local de realização da oficina}

As atividades da oficina de Higiene Pessoal foram executadas com crianças e adolescentes (Figura 1A) do Projeto Social Escolinha Tia More, localizado no bairro Jardim Canguru, no município de Campo Grande/MS. Adultos locais responsáveis pelo projeto e alguns pais também acompanharam a execução das atividades. A oficina de higiene pessoal foi desenvolvida por acadêmicos de Odontologia e Farmácia, sob a supervisão de professores do Instituto de Biociências da Universidade Federal de Mato Grosso do Sul (UFMS), Campo Grande.

\subsection{Atividades preparatórias}

A oficina foi primeiramente planejada e organizada por meio de duas reuniões semanais virtuais e/ou presenciais, período em que ocorreram pesquisas sobre o tema, seleção de atividades, aquisição de materiais e preparo dos acadêmicos ministrantes. Após as pesquisas referentes à temática da oficina, foram separadas algumas atividades lúdicas e interativas, que abordam os conhecimentos relativos aos cuidados de higiene pessoal. 
Abaixo encontram-se com mais detalhes os métodos utilizados para a realização com interatividade dos indivíduos que participaram do Projeto de Extensão de Ferramentas Socioambientais em Prol da Saúde:

\subsection{Atividades selecionadas para as oficinas}

A oficina foi realizada no dia 23 de outubro de 2020 com duração de 3 horas. Foram selecionadas as seguintes atividades, 1) apresentação de vídeos e canções com violão; 2) prática de lavagem das mãos; e 3) diálogo sobre a forma correta de tomar banho e cuidados necessários durante a COVID-19, descritos em detalhes, a seguir. Essas atividades foram realizadas com a participação de 17 crianças, faixa etária de 4 a 10 anos, e 3 adolescentes, na faixa etária de 12 a 14 anos.

\subsubsection{Apresentação de vídeos e canções com violão:}

A primeira atividade foi realizada por meio da projeção de dois vídeos. O primeiro vídeo foi da canção "Lavar As Mãos", composta por Arnaldo Antunes e disponível na plataforma de vídeos YouTube: https://www.youtube.com/watch?v= 6 8QfyCuqY. O segundo vídeo foi "Banho É Bom (Ratinho Tomando Banho)", composta por Hélio Zinskind e disponível também na mesma plataforma de vídeos em: https://www.youtube.com/watch?v=s9p9m0ebJmg. Após a exibição dos vídeos, as músicas foram tocadas no violão e cantadas com as crianças (Figura 1B). As temáticas envolvidas foram a lavagem das mãos e o banho, e durante as canções movimentos ilustrativos e adequados aos respectivos temas foram executados por todos (Anexo 1).

\subsubsection{Prática de lavagem das mãos:}

A atividade foi iniciada com a demonstração de como a sujeira se adere às mãos quando sujas, utilizando o experimento do orégano (Figura 1C) (Prefeitura Municipal de Chiapetta, 2020). Essa dinâmica foi realizada utilizando-se uma bacia com água e orégano e outra bacia com água e sabonete. A dinâmica se iniciou com uma breve explicação de que os microorganismos estão presentes 
na superfície das mãos, embora sejam invisíveis, e que precisamos lavar cuidadosamente as mãos para removê-los.

Assim, as crianças foram direcionadas, uma de cada vez, a colocarem as mãos na bacia com água e orégano (representando os micro-organismos) que gruda nas mãos. Em seguida, cada criança colocava a mão na bacia com sabonete simulando a lavagem das mãos, e então, ao retornar a mão à bacia com orégano, este era repelido. Ao final da dinâmica foi explicado, de maneira bem simples de modo a que todos compreendessem, que quando as mãos estão limpas, os micro-organismos são repelidos pelas mãos.

Em continuidade à prática de lavagem das mãos foi realizada uma outra atividade de pintura com as mãos com argila colorida. Nessa ação, as mãos ficavam sujas de argila colorida também representando as sujidades do ambiente. Em seguida, as crianças eram direcionadas à pia onde recebiam sabonete e aprendiam a como lavar as mãos de maneira correta (Anexo 1). Ao final, a mão de cada criança foi analisada para garantir que não houvesse resquícios de tinta. Caso houvesse, a criança era estimulada a lavar as mãos novamente como aprendeu.

\subsubsection{Diálogo sobre a forma correta de tomar banho e cuidados necessários durante a COVID-19:}

O diálogo sobre o banho foi realizado mostrando-se os itens necessários para sua execução, como: Água (chuveiro ou banheira), sabonete, shampoo e bucha (sintética ou vegetal) (Figura 1D). Cada item era apresentado para as crianças explicando como se utilizava da maneira correta. Na sequência, o tema sobre prevenção ao coronavírus foi trabalhado com cartazes e banners produzidos e cedidos pela Universidade Federal de Mato Grosso do Sul (Anexo 2). Foi demonstrado também a maneira correta do uso da máscara (Anexo 3).

Ao final de todas as atividades, foi realizada a distribuição de materiais de higiene pessoal que incluía: um recipiente contendo sabonete líquido, um sabonete em barra e uma bucha vegetal para cada participante. 

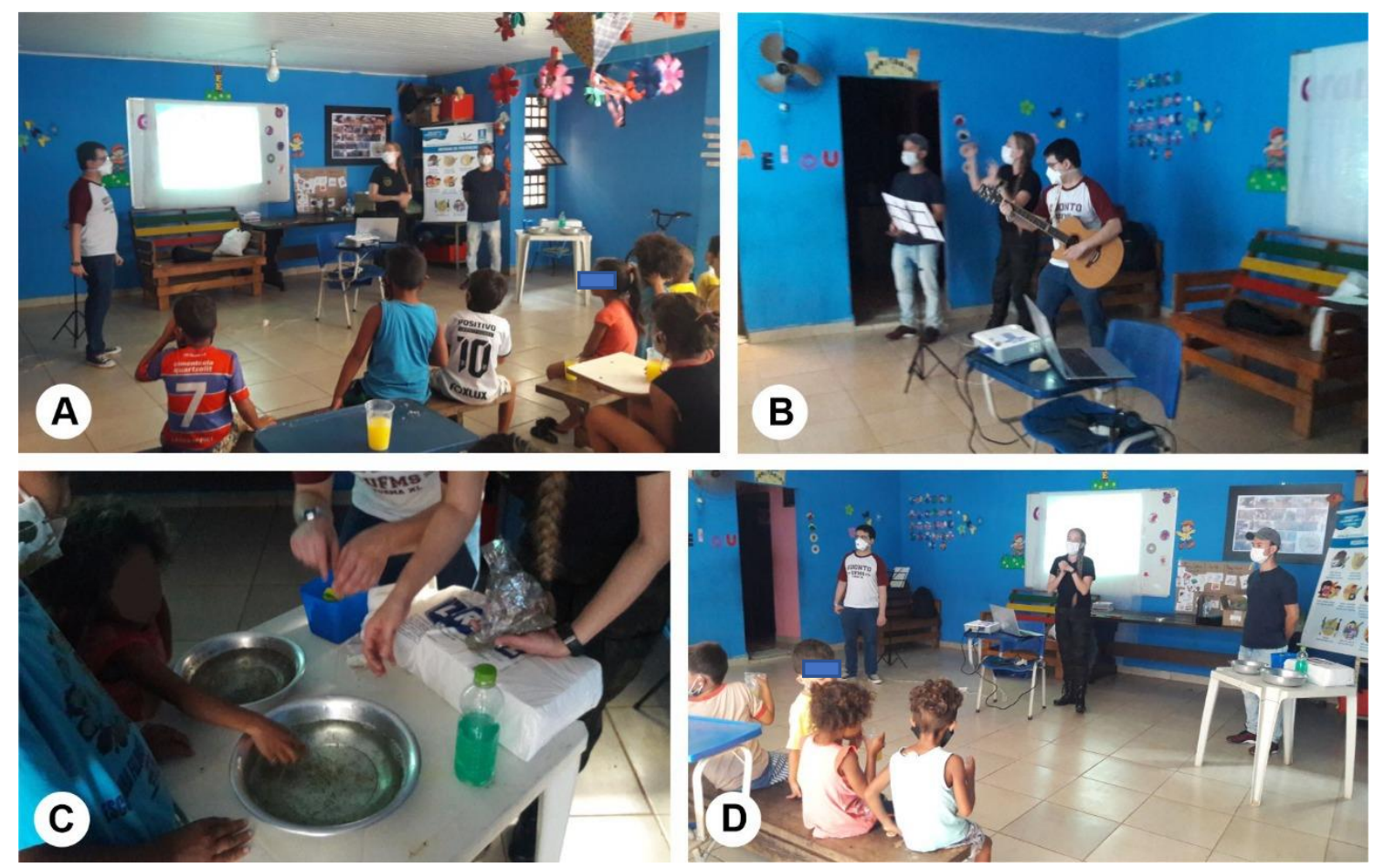

Figura 1: A - Desenvolvimento da Oficina de Higiene Pessoal no Projeto Social Escolinha Tia More, Campo Grande, MS. B - Atividade de canção com o violão. C Prática de lavagem das mãos, dinâmica usando bacia com água e orégano. D - Diálogo com as crianças e adolescentes sobre a forma correta de tomar banho.

\section{Resultados e Discussão}

Foram atendidos 20 pessoas, 17 crianças, na faixa etária de 4 a 10 anos, e 3 adolescentes, na faixa etária de 12 a 14 anos, alguns acompanhados de seus pais ou responsáveis, o que também se considerou positivo visto que eles desempenham um papel fundamental na educação dos filhos.

Em sua maioria, os participantes foram atenciosos e empenhados em aprender. Parte das crianças foi participativa respondendo às perguntas e interagindo durante as dinâmicas. Alguns, os mais tímidos, interagiram menos, mas ficaram atentos às atividades e as fizeram. As atividades que mais atraíram as crianças foram as de lavagem das mãos com a dinâmica do orégano, depois o uso da argila colorida associado à lavagem das mãos na sequência e cantar as letras das canções ao som do violão.

A dinâmica do orégano demonstrou resultado positivo ao despertar a atenção das crianças. Relacionar as folhas do orégano com micro-organismos invisíveis como bactérias e vírus foi primordial para facilitar a compreensão, 
sempre usando uma linguagem adequada à cada faixa etária. Essa atividade foi desenvolvida em escolas municipais de Chiapetta, RS, Brasil, como medida educativa de prevenção e de combate ao Coronavírus (Prefeitura Municipal de Chiapetta, 2020).

$\mathrm{Na}$ atividade com uso de argila colorida e de lavagem das mãos, foi notável a dificuldade das crianças em realizar a limpeza na região dos pulsos e das unhas, locais que em muitas crianças ainda havia tinta após a lavagem das mãos. Nessa experiência as crianças puderam novamente compreender e "enxergar" que micro-organismos invisíveis ainda ficam presentes embaixo de unha e dobras nas mãos. E então lavavam-se os resquícios de sujeira, reforçando, assim, a importância da adequada lavagem das mãos. O fato foi abordado de maneira divertida sem causar constrangimento às crianças.

Capacitar os indivíduos com informações expositivas e associadas a atividades lúdicas como recursos audiovisuais, sonoros e táteis são formas eficazes de se alcançar o foco das crianças e de se consolidar o seu aprendizado (CARDOSO; RODRIGUES, 2016), e assim as crianças conseguem aprender a separar de forma mais efetiva os objetos de suas significações. Portanto, esse mecanismo de edificar cenários estimula e possibilita uma relação maior com o contexto social, com o mundo real e com situações imaginárias, as quais são vistas como imprescindíveis para a aprendizagem.

Uma experiência semelhante ocorreu com acadêmicos do curso de Ciências Biológicas em Belo Horizonte, que por meio de práticas científicas e educativas atenderam uma população visando à mudança de comportamento para estimular a higiene como um hábito cotidiano, incluindo crianças e seus pais ou responsáveis. Foram desenvolvidos cartazes, cartilhas, jogos, peças de teatro, e distribuídos vários "Kits da Saúde" com escova de dente, pasta de dente e sabonetes. Foi também mencionada a importância de se utilizar vocabulário simples e adequado para a idade das crianças. Além disso, as atividades didáticas ajudam na fixação do conteúdo ensinado, e um momento final para revisão e perguntas foi visto como fortalecedor desse processo (Ferreira, 2008).

Durante a realização da oficina observamos boa interação das crianças e adolescentes com os membros da equipe, fato que favoreceu ainda mais a comunicação durante as atividades. Além disso, a observação do interesse das 
crianças nas atividades foi importante para notar a necessidade de tornar as atividades mais dinâmicas, interessantes e interativas. Quando se julgava necessário, trazia-se mais perguntas ao invés de somente dar explicações, e isso se mostrou muito eficaz.

A limitação do estudo foi em relação a proximidade social e física com os participantes, que a pandemia do COVID-19 proporciona, foi uma barreira ao acolhimento. Porém, a interatividade despertou a curiosidade e o engajamento nas crianças, não só na apresentação expositiva, mas principalmente nas músicas de violão e na prática de lavagem de mãos, que foram atividades bem positivas.

\section{Conclusão}

Ressalta-se que o desenvolvimento da oficina foi relevante para atenuar a ação cotidiana de determinantes sociais do processo saúde-doença sobre as crianças e famílias moradoras do bairro em situação de vulnerabilidade social. A região apresenta acesso ao saneamento básico e à Unidade Básica de Saúde (UBS) em suas proximidades, o que é algo positivo para a qualidade de vida da comunidade.

Conclui-se que os objetivos da oficina foram alcançados com sucesso. Ao final, as crianças e adolescentes receberam os materiais do kit de higiene pessoal como medida para reforçar e estimular os hábitos recém adquiridos, e com isso implementá-los no cotidiano de cada uma das pessoas envolvidas. Quando questionados sobre o uso dos materiais todos se mostraram entusiasmados com o conhecimento adquirido.

Nota-se também, que a interatividade com o público reforça o aprendizado, uma vez que estimula o conhecimento e maior adesão para o-médio e longo prazo. Alguns participantes responderam às perguntas, afirmando conhecerem as práticas básicas de banho e lavagem de mãos.

Espera-se que este relato de experiência incentive outras instituições e formadores brasileiros a desenvolverem esse tipo de ação, com o intuito de reforçar os hábitos de higiene pessoal, contribuindo para a prevenção de doenças e a promoção de qualidade de vida por meio da educação em saúde. 


\section{Referências}

BORTOLOTTO I. M. et al. A knowledge network to promote the use and valorization of wild food plants in the Pantanal and Cerrado, Brazil. Regional Environmental Change, v. 17, p. 1329-1341, 2016. https://doi.org/10.1007/s10113-016-1088-y. Disponível em: https://link.springer.com/article/10.1007/s10113-016-1088-y\#citeas. Acesso em: fev 2021

BRASIL. Portaria n 5ㄴ de 11 de março de 2020. Dispõe sobre a regulamentação e operacionalização do disposto na Lei no 13.979, de 6 de fevereiro de 2020, que estabelece as medidas para enfrentamento da emergência de saúde pública de importância internacional decorrente do coronavírus (COVID-19). Diário oficial da união, Ministério da Saúde, Brasília, DF, 12 mar. 2020. Seção 1, p. 185.

BRASIL. [Constituição (1988)]. Constituição da República Federativa do Brasil de 1988. Brasília, DF: Presidência da República. Disponível em:

http://www.planalto.gov.br/ccivil_03/constituicao/constituicao.htm. Acesso em: 5 mar. 2021.

CAlvacANTE-NETO, A. S.; OliveirA, M. A. C; BeZerRA, E. A. Plano de Contingência Contra O COVID-19: Análise Crítica. Revista Saúde e Meio Ambiente RESMA, v. 11, n. 2, p. 373-386, ago./dez., 2020.

CAMPO GRANDE NEWS. Empresa vai avaliar casas que abrigam ex-moradores da Cidade de Deus. CAMPO GRANDE NEWS, 2017. Disponível em: https://www.campograndenews.com.br/cidades/capital/empresa-vai-avaliar-casas-queabrigam-ex-moradores-da-cidade-de-deus. Acesso em: 05 mar. 2021.

CAMPO GRANDE NEWS. Rede de esgoto chegou a 77\% da Capital nos últimos 5 anos. Porém, pesquisa mostra que atualmente $58,38 \%$ dos dejetos na cidade são tratados. CAMPO GRANDE NEWS, 2008.2 Disponível em: https://www.campograndenews.com.br/meio-ambiente/rede-de-esgoto-chegou-a-77da-capital-nos-ultimos-5-anos. Acesso em: 05 mar. 2021.

CARDOSO, S. M. M; RODRIGUES, A. P. Promoção da saúde a partir das demandas relacionadas à higiene e saúde na escola. Revista de Ciência e Inovação do IF Farroupilha, v. 1, n. 2, p. 93-104, 2016. 
FARIAS H. S. O avanço da Covid-19 e o isolamento social como estratégia para redução da vulnerabilidade, Espaço e Economia [Online], v. 17, 2020. Disponível em: http://journals.openedition.org/espacoeconomia/11357. Acesso em: 05 mar. 2021.

FERREIRA, E. A. Projeto Educação e Saúde: a importância das medidas de higiene para a profilaxia de doenças parasitárias. 2008. 15 p. Trabalho de Conclusão de Curso (Graduação em Ciências Biológicas) - Centro Universitário Metodista Izabela Hendrix, Belo Horizonte.

FERRIANI, M. G. C.; GOMES, R. Saúde Escolar - Contradições e Desafios. 1. ed. Goiânia: AB Editora, 1997. ix, 63 p.

IBGE, 2019. Extrema pobreza atinge 13,5 milhões de pessoas e chega ao maior nível em 7 anos. Rio de Janeiro, 06 nov. 2019. Disponível em: https://agenciadenoticias.ibge.gov.br/agencia-noticias/2012-agencia-denoticias/noticias/25882-extrema-pobreza-atinge-13-5-milhoes-de-pessoas-e-chega-aomaior-nivel-em-7-anos. Acesso em: 08 fev. 2021.

KHATOON, R. et al. Impact of school health education program on personal hygiene among school children of Lucknow district. Journal of Family Medicine and Primary Care, v. 6, n. 1, p. 1-3, 2017. 10.4103/2249-4863.214973. Disponível em: . Acesso em: 05 mar. 2021

LEME F.M. et al. Ensino de botânica e educação ambiental: modelos didáticos e oficinas pedagógicas. Educação Ambiental em Ação, v. 1, n. 53, 2015. Disponível em: http://revistaea.org/artigo.php?idartigo=2122. Acesso em: mar de 2021.

LEONETI, A. B.; PRADO, E. L. DO; OLIVEIRA, S. V. W. B. DE. Saneamento básico no Brasil: considerações sobre investimentos e sustentabilidade para o século XXI. Revista de Administração Pública, v. 45, n. 2, p. 331-348. 2011.

OLIVEIRA, A. C.; LUCAS, T. C.; IQUIAPAZA, R. A. O Que A Pandemia da COVID-19 Tem Nos Ensinado Sobre Adoção de Medidas de Precaução? Texto \& Contexto Enfermagem, v. 29, n. 106, p. 4- 6, 2020. https://doi.org/10.1590/1980-265x-tce-20200106. Disponível em: https://www.scielo.br/scielo.php?script=sci arttext\&pid=S010407072020000100201\&tlng=en. Acesso em: fev 2021. 
PREFEITURA MUNICIPAL DE CHIAPETTA. Atividades lúdicas nas escolas ensinam como se prevenir e evitar o contágio do Coronavírus. Chiapetta, 20 set. 2020. Disponível em: http://chiapetta.rs.gov.br/2020/03/19/atividades-ludicas-nasescolas-ensinam-como-se-prevenir-e-evitar-o-contagio-do-coronavirus/. Acesso em: 20 dez. 2020.

ROCHA, G. B. A.; SILVA, B. G. B. A Importância de Estimular Os Hábitos de Higiene Pessoal na Educação Infantil. Revista Educação e (Trans)formação, p. 19-30, 2018. Disponível em: http://www.journals.ufrpe.br/index.php/educacaoetransformacao/article/view/2056. Acesso em: mar de 2021.

SOUZA-NETO, J. C.; CARVALHO FILHO, D. M.; MARTINS, A. C. M. Hábitos de Higiene na Infância: Relato de Experiência na Comunidade São Rafael. In: Silva Neto, B. R. Da (org.). Medicina: Elevados Padrões de Desempenho Técnico e Ético, Atena Editora, v. 3, n. 1800 , p. 69-72, 2020.

TEIXEIRA, T. A.; FANTINATTI, M; GONÇALVES, M. P.; SILVA, J. S. DA. Parasitoses intestinais e saneamento básico no Brasil: estudo de revisão integrativa. Brazilian Journal of Development v. 6, n. 5, p. 22867-22890. 2020.

WIRTI, D. H.; BERNARDELLI, M. L. F. H. A Favela Cidade de Deus em Campo Grande - MS: gênese, conflitos e a negação do direito à moradia. Estudos Geográficos, Rio Claro, v. 14, n. 2, p. 70-94. 2016. Disponível em: http://www.periodicos.rc.biblioteca.unesp.br/index.php/estgeo. Acesso em: 05 mar. 2021.

ZHOU, P. et al. A pneumonia outbreak associated with a new coronavirus of probable bat origin. Nature, v. 579, p. 270-273, 2020. https://doi.org/10.1038/s41586-020-20127 Disponível em:https://www.nature.com/articles/s41586-020-2012-7. Acesso em: 05 mar. 2021.

ZHU, N. et al. A Novel Coronavirus from Patients with Pneumonia in China. The New England Journal of Medicine, v. 382, 727-733. 2019. DOI: 10.1056/NEJMoa2001017. Disponível em: https://www.nejm.org/doi/10.1056/NEJMoa2001017. Acesso em: 05 mar. 2021. 


\section{Agradecimentos}

Os autores agradecem a coordenadora, Edileuza Luiz, do Projeto Social Escolinha Tia More, por abrir as portas para o desenvolvimento das atividades da ação de extensão e a Universidade Federal de Mato Grosso do Sul/UFMS por fornecer recursos para a execução da ação, por meio do Edital PROECE/UFMS № 43 de março de 2020 (PAEXT/2020). 